\title{
Negative effects of makeup use on perceptions of leadership ability across two ethnicities
}

\author{
ESTHER A JAMES, SHAUNY JENKINS \& \\ CHRISTOPHER D WATKINS*
}

James, E. A., Jenkins, S., \& Watkins, C. D. (2018). Negative effects of makeup use on perceptions of leadership ability across two ethnicities. Perception. DOI:

$10.1177 / 0301006618763263$

Reprinted by permission of SAGE Publications

*Corresponding Author: Dr Christopher D Watkins Division of Psychology, School of Social and Health Sciences Abertay University Bell Street, Dundee DD11HG 


\section{Negative effects of makeup use on perceptions of leadership ability across two ethnicities}

Cosmetics alter social perceptions, and prior work suggests that cosmetic use may aid female intrasexual competition, making women appear more dominant to other women but more prestigious to other men. It is unclear whether these findings reflect general improvements in perceptions of traits related to women's dominance or if they are specific to mating contexts only. Here, across two ethnicities, we examined

effects of cosmetics used for a social night out on perceptions of women's leadership ability, a trait that denotes competence/high status outside of mating contexts.

Participants of African and Caucasian ethnicity judged faces for leadership ability where half of the trials differed in ethnicity (own- versus other-ethnicity face pairs) and the subtlety of the colour manipulation (50\% versus $100 \%)$. Regardless of the participant's sex or ethnicity, makeup used for a social night out had a negative effect on perceptions of women's leadership ability. Our findings suggest that, in prior work, women are afforded traits related to dominance as makeup enhances perceptions of traits that are important for successful female mating competition but not other components of social dominance such as leadership.

Keywords: Adornment, cosmetics, dominance, intrasexual competition, facial coloration 


\section{Introduction}

Cosmetics use has a long history (Ungar et al., 2002) and, as adornments, cosmetics may be part of the extended phenotype (Etcoff et al., 2011) and important in the study of gene-culture coevolution. Indeed, consistent with the proposed cultural importance of adornments to manipulate cues to reproductive fitness, preferences for purchasing such items strengthen when resources are scarce, in contrast to spending on other luxury consumer products (Hill et al., 2012). Sexual selection theories of human mate preferences suggest that attractiveness judgements reflect an underlying 'quality' of the beholder (e.g., Gangestad \& Scheyd, 2005; Little et al., 2011). Consistent with this, natural and artificial changes to skin or lip coloration are associated with cues to mate quality such as health, youth, fertility and attractiveness (Foo et al., 2017; Jones \& Kramer, 2016; Jones et al., 2016; Stephen \& McKeegan, 2010; Stephen et al., 2009; see also Henderson et al., 2016 for a review). Moreover, makeup alters the contrast between different facial features (Russell, 2009) and does so across different cultures (Jones et al., 2015). These changes to contrast are negatively related to perceptions of age (Porcheron et al., 2013; Russell et al., 2017), even when assessed cross-culturally (Porcheron et al., 2017). In addition, facial contrast enhances judgements of both health (Russell et al., 2016) and attractiveness (Russell, 2003). Collectively, cosmetics and their effects on skin coloration are important in human mate choice.

Makeup application may also have corresponding effects on judgements that are important for successful resource competition (i.e. identifying traits related to dominance in others; Mileva et al., 2016). Complementing work on relationships between attractiveness and earnings (Hammermesh \& Biddle, 1993), makeup use is related to tip earnings (Jacob et al., 2009) and, conversely, lack of makeup is 
associated with 'thin-slice' judgements of having a low status job (Nash et al., 2006). People appear to make implicit associations between makeup and status (Richetin et al., 2004) and, in images of female models, makeup enhances perceptions of competence (Etcoff et al., 2011), complementing other work which suggests potential 'halo effects' ascribed to women with makeup such as 'organized' (Graham \& Jouhar, 1981), bright and assertive (Osborn, 1996). While these findings suggest evidence for relationships between social dominance and makeup in women, no work to our knowledge has directly tested whether makeup enhances perceptions of leadership ability. In recent work, makeup enhances women's attractiveness to other men and women but enhances perceptions of women's dominance among female judges only, while enhancing perceptions of women's prestige among male judges only (Mileva et al., 2016). Consistent with the importance of physical attractiveness in female intrasexual competition (reviewed in Vaillancourt, 2013), these sex differences in social attributions were driven by female jealousy of potential samesex rivals for mates when they were wearing makeup (Mileva et al., 2016). Collectively, makeup use may be important for both mate choice and intrasexual competition.

The current study extends research on social responses to makeup. First, we test whether we observe similar sex differences in social responses to makeup when individuals are specifically asked to judge women with versus without makeup on their ability to lead others (one component of social dominance). Mileva and colleagues asked participants to rate faces on traits related to dominance without defining the traits (dominance and prestige) to participants. While this procedure is common in research on social judgements of faces, directly testing for effects of makeup on perceived leadership ability enables us to establish whether traits related 
to dominance that are conferred upon women with makeup generalize to aspects of dominance where competence and intelligence are valuable (i.e. leadership; Todorov et al., 2005, 2015). Alternately, we can clarify whether prior findings in this area reflect some other unidentified component of dominance, most likely the prestige freely conferred by men onto an attractive woman and, in turn, women's gauge of other women's effectiveness as competitors for mates (see Vaillancourt, 2013 for a review).

Second, we test whether Mileva et al.'s (2016) findings generalize across ethnicities. Research on responses to facial coloration suggest that judgements of own-race faces that are important for mate choice generalize to other-race faces when manipulated along the same colour dimension (Stephen et al., 2011). However, group competition is salient in the evolution of leadership roles (van Vugt et al., 2008; see also Choi \& Bowles, 2007). Moreover, interactions between groups are more competitive than interactions between individuals (Wildschut et al., 2003) and cues to out-group membership can promote negative social evaluations of others (e.g., Blair et al., 2004; see also Dotsch et al., 2008). Thus, we test whether leadership evaluations of women with makeup differ (i.e. are stronger or weaker) or are identical for 'out-group' faces (African or Caucasian) versus in-group (own ethnicity) faces.

Finally, we manipulate the subtlety of our colour manipulation in order to test whether this alters evaluations of leadership ability. This extends Mileva and colleagues' work, by taking into account evidence that women wear more makeup than is optimally attractive (Jones et al., 2014), and that quantity of makeup alters perceptions of traits that may be important in a good leader, such as likeability, trustworthiness and competence (Etcoff et al., 2011). If, in general, makeup 
enhances perceived leadership ability, this would be consistent with prior work relating makeup to status (reviewed in Mileva et al., 2016). Alternately, if makeup weakens perceived leadership ability, we would extend prior work, which demonstrates that makeup enhances perceptions of traits related to dominance when competing for mates, by suggesting that these patterns of findings do not generalize to leadership roles where women compete for other resources.

\section{Method}

\section{Participants}

One hundred sixty-eight individuals (48 of whom were male, Mage=23.25 years, $S D=4.87$ years: 29 Caucasian males, 47 Caucasian females, 19 males of African ethnicity, 73 females of African ethnicity) who self-reported their ethnicity took part in the online face perception task hosted on surveymonkey.com. Participants of African ethnicity were primarily British and Irish participants of African descent. Responses from the same device were not permitted. Online and laboratory experiments on social judgements of faces produce equivalent results (e.g., Watkins, 2017; Wilson \& Daly, 2004; see also Gosling et al., 2004 for a review). Our sample size exceeds $90 \%$ power to detect moderate effects (via follow-up paired t tests), both across the sample, within a given ethnicity (of rater) and within a given gender (of rater).

\section{Face stimuli}

Caucasian prototype constituents. In a separate study run by the second author (a trained makeup artist), three young white-British females (Mage=18.57 years, $S D=0.11$ years) were recruited to generate two prototype female faces representing the average colour features of the same women before and after a makeover. 
Women were photographed using a Panasonic Lumix DMC-G3 in a standardized setup (constant lighting, soft box lighting, constant white backdrop and camera position). Women came to the laboratory without makeup and were asked to remove all adornments and to pose with a neutral expression with direct gaze and hair tied back from forehead. Participants had their photograph taken twice (i.e. before and after their makeover) in the same manner. During the makeover, the researcher used her professional judgement as a makeup artist to apply makeup that would be suitable for a social night out (sensu Mileva et al., 2016). Software was then used to average the colour features of the three female faces, generating a before and after prototype of the same three women (i.e. two separate face prototypes). Images were standardized on pupil position, resized to $300 \times 400$ pixels and were masked to remove hair and background cues to that only the face, neck and ears were visible (see Figure 1).

In a pilot study, the two facial composites were rated by a separate panel of judges for attractiveness, with each participant indicating their preferred face in the pair and the extent to which they preferred their chosen face relative to the alternate face. Ratings were coded using the scale 0-3 (No makeup prototype: 'much more attractive' (=0), 'more attractive' (=1), 'somewhat more attractive' (=2), 'slightly more attractive' (=3) than makeup prototype) and 4-7 (Makeup prototype: 'slightly more attractive' (=4), 'somewhat more attractive' (=5), 'more attractive' (=6), 'much more attractive' (=7) than no makeup prototype). Across participants, ratings of the facial composites (97 participants, 44 of whom were male: Mage=26.81 years, $S D=7.85$ years) revealed that the makeover enhanced attractiveness in the expected direction (Mattractiveness=4.76, SEM=.17, $t(96)=7.40 ; p<.001, r=0.35)$. 
African prototype constituents. In a separate initial session run by the first author, three women of African ethnicity (Mage=20 years, $S D=1.0$ years) followed the same procedure as above except that they had their photograph taken before and after they self-applied makeup that they would be comfortable with wearing on a social night out. The women photographed here were a mixture of African women and western women of African descent. These photographs were used to generate two face prototypes, representing the average colour features of the same three women of African ethnicity before their makeover and, separately, after their makeover. Women were photographed under the same conditions as other women who provided their image for later manipulation in this study.

Manipulated faces. Individual face images were manipulated in colour cues related to cosmetic use by manipulating a separate group of four Caucasian women's faces $\left(M_{\mathrm{age}}=21.58\right.$ years, $S D=2.29$ years $)$ and four African women's faces $\left(M_{\mathrm{age}}=21\right.$ years, $S D=0.82$ years). The Caucasian women who provided images took part in a separate laboratory project unrelated to the current experiment, however all participants were tested in the same laboratory under the same photographic conditions as the constituents of the prototypes. Image donors were recruited as a sample of convenience by the first author (women of African ethnicity) and second author (Caucasian women).

For the colour manipulation, prototype based image transformation was used (e.g., Perrett et al., 1998) to manipulate facial colouration only and generate two different versions of the same identity. Two different colour transformations were applied for each identity, resulting in a (more subtle) $50 \%$ colour manipulation and a $100 \%$ colour manipulation of the same woman. All images were standardized on 
pupil position, resized to $300 \times 400$ pixels and were masked to remove hair and background cues to that only the face, neck and ears were visible (see Figure 1).

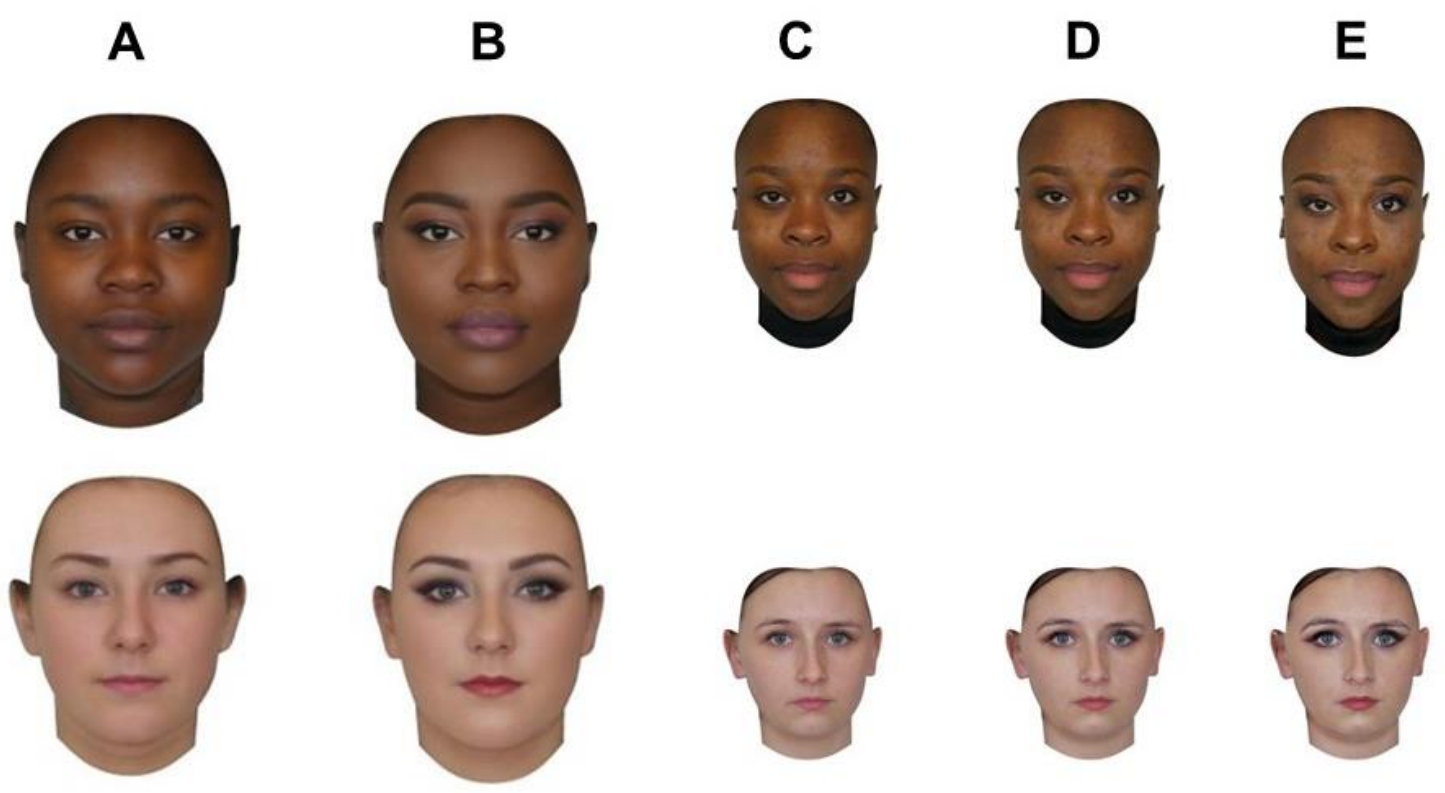

Figure 1. The average colour characteristics of women of Caucasian and African ethnicity before $(A)$ and after (B) a makeover for a social night out. Transforming individual women without makeup (C) to generate a subtle (50\%) makeup manipulation (D) and a 100\% makeup manipulation (E).

\section{Procedure}

Each participant completed a sixteen-trial face perception task where they judged eight Caucasian face-pairs and eight African face-pairs for leadership ability. Within these pairs, eight of the pairs consisted of a makeup-free woman versus a $50 \%$ colour manipulation of that woman and the other eight pairs consisted of the same identities without makeup versus a $100 \%$ colour manipulation of that woman. Trials were presented in a randomized order with labels 'Image a' and 'Image b' above left and right face respectively. The side of the screen on which the face without makeup appeared was counterbalanced across trials. On each trial, participants were asked to indicate which face they judged to be the better leader and how much better they judged their chosen face to be relative to the other face in the pair. 


\section{Initial processing of data}

Responses to the face perception task were coded:

0-3: Face without makeup judged 'much better' (=0), 'better' (=1), 'somewhat better' (=2), 'slightly better' (=3) leader than the face with makeup.

4-7: Face with makeup judged 'slightly better' (=4), 'somewhat better' (=5), 'better' $(=6)$, 'much better' $(=7)$ leader than the face without makeup.

Responses to our face perception task were averaged across trials, separately, for own-ethnicity trials and other-ethnicity trials and separately for 50\% makeup trials and $100 \%$ makeup trials (i.e. to examine the within subjects factors face ethnicity and makeup level). High scores on our task represent a stronger tendency to associate colour cues related to makeup with leadership ability.

\section{Results}

A repeated-measures ANOVA was conducted on the dependent variable perceived leadership ability, with the factors face ethnicity (own-ethnicity, other-ethnicity), makeup level (50\% manipulation, 100\% manipulation) and participant sex (male, female). This analysis revealed a main effect of makeup level $(F(1,166)=11.01$; $p=.001, \mathrm{np} 2=.06$ ) and no other significant effects or interactions (all $F<2.52$ all $p>$.12). Paired t tests to interpret this effect revealed that the $100 \%$ colour manipulation had a negative effect on perceived leadership ability $(M=3.24$, $S E M=.08)$ compared to the $50 \%$ colour manipulation $(M=3.59$, $S E M=.06$, $t(167)=4.04 ; p<.001, r=0.31)$. Follow-up analyses confirmed that while the $100 \%$ colour manipulation weakened perceived leadership ability at levels that differed 
from chance (absolute $t(167)=3.42 ; p=.001, r=0.13$, see Figure 2 ), the $50 \%$ colour manipulation did not alter perceived leadership ability at levels that differed from chance (i.e. 3.5 , absolute $t(167)=1.48 ; p=.14, r=0.05$ ).

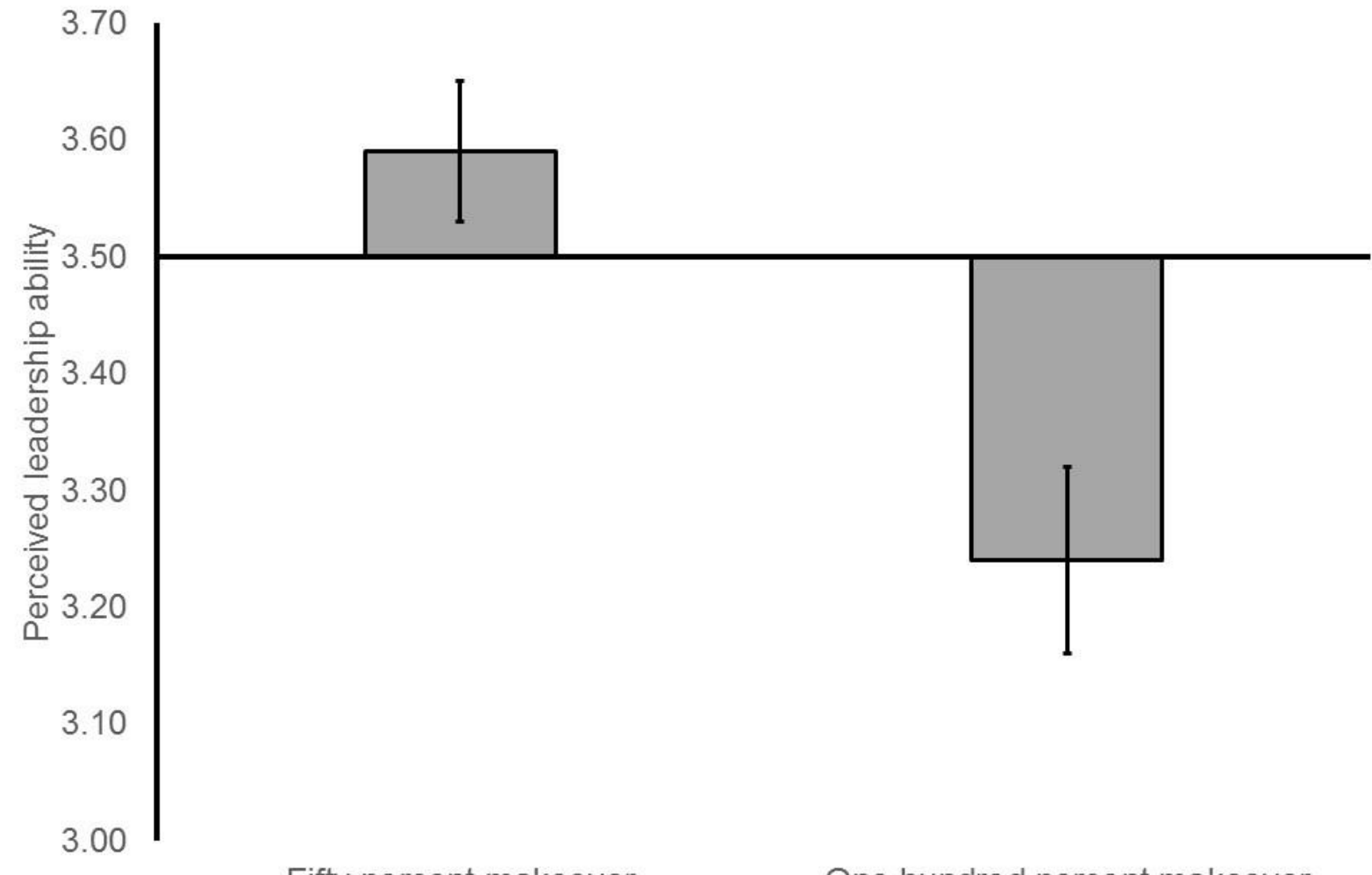

Fifty percent makeover One hundred percent makeover

Figure 2. Negative effects of makeup manipulation on perceived leadership ability, regardless of the sex or ethnicity of the judge (effect size $r=0.13$ ).

\section{Discussion}

Our findings extend recent research on social judgements of makeup (Mileva et al., 2016) by demonstrating that makeup has negative effects on perceptions of women's leadership ability. Moreover, these findings were consistent across male and female judges, and when individuals judged both own-ethnicity and otherethnicity faces manipulated in colour cues to makeup. Of note, our colour 
manipulation in African faces was generated by asking participants to self-apply makeup for a social night out (sensu Mileva et al., 2016) and the corresponding manipulation in Caucasian faces was generated by the application of makeup for a social night out from a trained makeup artist (the second author). Thus, our data also suggest that negative effects of makeup on perceived leadership ability are consistent regardless of the makeover process used to generate prototypes for manipulating individual face images. Our findings therefore complement prior work on perceptions of colour cues in the face across ethnicities (e.g., Jones et al., 2015; Porcheron et al., 2017) by suggesting that colour manipulations generalize across ethnicities when examining their corresponding effects on judgements important for mate choice and, in the current study, perceptions of leadership ability.

Our findings develop work by Mileva and colleagues (2016) as our data suggest that their prior work, where women were afforded traits related to dominance when wearing makeup, is likely due to makeup enhancing their perceived effectiveness as a competitor for a male mate (i.e., attractive women; Vaillancourt, 2013) rather than perceptions of their ability to lead others. Although prior work associates makeup with social dominance (see Mileva et al., 2016), positive effects of makeup may be specific to certain roles within an organization, such as in service industry roles where attractiveness to clientele may be more important for eliciting prosocial responses (e.g., from customers via tipping; Jacob et al., 2009). Indeed, prior work suggests that makeup may have negative effects on judgements of performance depending on whether the position under evaluation is a stereotypically female occupation (Cox \& Glick, 1986). Our findings also complement recent work suggesting that traits derived from facial cues such as attractiveness may be 'valued' more at lower versus upper-level roles within an organization such as in retail 
(Fruhen et al., 2015). Competence (i.e., intelligence) is an important trait-dimension in leadership (Todorov et al., 2005) and our data suggest that makeup use has a negative effect on judgements of leadership. Further work could use data-driven methods to establish the various routes toward female status, and how traits related to female dominance relate to both their (natural) physical appearance and women's desire to enhance or alter their appearance.

Although not significantly different from chance, our subtle colour manipulation had a beneficial effect on perceived leadership ability relative to our $100 \%$ colour manipulation. Thus, we provide preliminary evidence that a subtle enhancement in facial colouration via makeup may have positive effects on how women are evaluated within the business environment. This factor may account for differences between our data, where makeup weakens perceptions of leadership ability, and prior work, which demonstrates a possible link between makeup and social status, albeit across studies that use different stimuli and methods (Etcoff et al., 2011; Jacob et al., 2009; Nash et al., 2006; Richetin et al., 2004). For example, further work could extend Etcoff et al. (2011), who examined judgements of female models, by altering the intensity of makeup application to test for its effects on judgements of women on other trait dimensions. As identity has stronger effects on social perceptions of women's faces than makeup application by those same women (Jones \& Kramer, 2015), and attractive women benefit less from makeup than their less-attractive peers do (Jones \& Kramer, 2016), further work could also test for interactions between facial attractiveness and makeup application on perceptions of traits related to dominance. Such work may prove fruitful, to test whether makeup application has stronger (positive or negative) effects on trait-evaluations of women who are 'naturally' more versus less attractive. The size of our stimulus set suggests 
that the broad generalizability of our findings to other individuals may be limited. However, effects of the intensity of our manipulation on judgements of leadership ability by the same raters (i.e. a repeated measures design), across the same identities and two different ethnicities, suggests that colour cues denoting quantity of makeup influence social judgements of women on this particular dimension.

There are a few additional points for discussion in light of our study design and findings. Following prior methods (Mileva et al., 2016), women applied makeup for a social night out in order to test whether the effects found in prior work generalize to other components of social dominance such as leadership ability. Although prior work suggests that women self-apply more makeup than is optimally attractive (Jones et al., 2014) and our stronger colour manipulation had greater negative effects on perceptions of leadership ability, further work could directly test whether self-application of makeup for different contexts (e.g. work versus a social night out) has differential effects on perceptions of women when judging them as leaders versus other traits related to dominance that may be more applicable to mating contexts. This could be fruitful, in light of recent work, which suggests that women distinguish between enhancing appearance for mate choice versus making a professional impression in the workplace (Netchaeva \& Rees, 2016).

Of note, our manipulation had negative effects on perceptions of leadership ability regardless of the ethnicity of the face under consideration, which runs counter to our theorizing that cues to out-group membership may moderate social judgements of faces on this trait dimension (i.e. stronger negative effects of makeup on perceptions of 'out-group' faces). This null finding may be accounted for, in part, simply by visual exposure to various ethnicities among our sample, which rendered ethnicity a weak cue to out-group membership. Our ideas could be tested further in 
ethnically homogenous samples, using arbitrary cues to group membership such as text-based labels, or by increasing the salience of between-group competition via experimental priming techniques.

Finally, the colour manipulation in the current experiment involves colour changes to large regions such as the lips or eyes but does not consider the role of other makeup such as foundation, which would influence perceptions of skin evenness. As colour changes to regions such as the lips can have corresponding effects on judgements related to attractiveness (Guéguen, 2012; Stephen \& McKeegan, 2010), our findings are still consistent with the proposal that colour changes related to makeup have corresponding negative effects on a specific component of female social dominance (i.e. leadership). In addition, these effects are stronger when the corresponding manipulation is more apparent to the observer. Indeed, if other forms of makeup have corresponding effects on facial contrast and judgements of traits related to women's attractiveness (e.g., Jones et al., 2015; Porcheron et al., 2017), our findings for effects of a colour manipulation on specific facial regions may represent an underestimate of the true (negative) effect of makeup on perceptions of her leadership ability.

To conclude, our work presents new evidence that colour cues related to makeup have negative effects on perceptions of women's leadership ability and that these evaluations are consistent among male and female judges and when judging faces of Caucasian and African ethnicity.

\section{Acknowledgements}

We thank the donors who volunteered their time during our makeover sessions. 


\section{Access to research materials}

Data can be accessed on request from the Corresponding Author.

\section{References}

Blair, I.V., Judd, C.M. \& Chapleau, K.M. (2004). The influence of afrocentric facial features in criminal sentencing. Psychological Science, 15, 674-679.

Choi, J. K. \& Bowles, S. (2007). The coevolution of parochial altruism and war. Science, 318, 636-640.

Cox, C. L. \& Glick, W. H. (1986). Resume evaluations and cosmetics use: When more is not better. Sex Roles, 14, 51-58.

Dotsch, R., Wigboldus, D. H. J., Langner, O. \& van Knippenberg, A. (2008). Ethnic out-group faces are biased in the prejudiced mind. Psychological Science, 19, 978-980.

Etcoff, N. L., Stock, S., Haley, L. E., Vickery, S. A. \& House, D. M. (2011). Cosmetics as a feature of the extended human phenotype: Modulation of the perception of biologically important facial signals. Plos One, 6, e25656.

Foo, Y. Z., Rhodes, G. \& Simmons, L. W. (2017). The carotenoid beta-carotene enhances facial colour, attractiveness and perceived health, but not actual health, in humans. Behavioral Ecology, 28, 570-578.

Fruhen, L. S., Watkins, C. D. \& Jones, B. C. (2015). Perceptions of facial attractiveness, dominance and trustworthiness predict managerial pay awards in experimental tasks. Leadership Quarterly, 26, 1005-1016.

Gangestad, S. W. \& Scheyd, G. J. (2005). The evolution of human physical attractiveness. Annual Review of Anthropology, 34, 523-548.

Gosling, S. D., Vazire, S., Srivastava, S. \& John, O. P. (2004). Should we trust webbased studies? A comparative analysis of six preconceptions about internet questionnaires. American Psychologist, 59, 93-104.

Guéguen, N. (2012). Does red lipstick really attract men? An evaluation in a bar. International Journal of Psychological Studies, 4, 206-209.

Hammermesh, D. S. \& Biddle, J. E. (1994). Beauty and the labor market. American Economic Review, 84, 1174-1194.

Henderson, A. J., Holzleitner, I. J., Talamas, S. N. \& Perrett, D. I. (2016). Perception of health from facial cues. Philosophical Transactions of the Royal Society $B$, 371, 1-9.

Hill, S. E., Rodeheffer, C. D., Griskevicius, V., Durante, K. \& White, A. E. (2012). Boosting beauty in an economic decline: Mating, spending, and the lipstick effect. Journal of Personality and Social Psychology, 103, 275-291.

Jones, A. L. \& Kramer, R. S. S. (2016). Facial cosmetics and attractiveness: Comparing the effect sizes of professionally-applied cosmetics and identity. PLoS One, 11: e0164218.

Jones, A. L. \& Kramer, R. S. S. (2015). Facial cosmetics have little effect on attractiveness judgements compared with identity. Perception, 44, 79-86.

Jones, A., Kramer, R. \& Ward, R. (2014). Miscalibrations in judgements of attractiveness with cosmetics. The Quarterly Journal of Experimental Psychology, 67, 2060-2068. 
Jones, A., Russell, R. \& Ward, R. (2015). Cosmetics Alter Biologically-Based Factors of Beauty: Evidence from Facial Contrast. Evolutionary Psychology 13. doi:10.1177/147470491501300113

Jones, A. L., Sweda, J. R., Porcheron, A. \& Russell, R. (2016). Coloration in different areas of facial skin is a cue to health: The role of cheek redness and periorbital luminance in health perception. Body Image. doi: 10.1016/j.bodyim.2016.02.001

Little, A. C., Jones, B. C. \& DeBruine, L. M. (2011). Facial attractiveness: Evolutionary based research. Philosophical Transactions of the Royal Society $B, 366,1638-1659$.

Mileva, V. R., Jones, A. L., Russell, R. \& Little, A. C. (2016). Sex differences in the perceived dominance and prestige of women with and without cosmetics. Perception, 45, 1166-1183.

Nash, R., Fieldman, G., Hussey, T., Leveque, J. L. \& Pineau, P. (2006). Cosmetics: They influence more than Caucasian female facial attractiveness. Journal of Applied Social Psychology, 36, 493-504.

Netchaeva, E. \& Rees, M. (2016). Strategically stunning: The professional motivations behind the lipstick effect. Psychological Science, 27, 1157-1168.

Perrett, D.I., Lee, K.J., Penton-Voak, I.S., Rowland, D.R., Yoshikawa, S., Burt, D.M., Henzi, S.P., Castles, D.I. \& Akamatsu, S. (1998). Effects of sexual dimorphism on facial attractiveness. Nature, 394, 884-887.

Porcheron, A., Mauger, E., Soppelsa, F., Liu, Y., Ge, L., Pascalis, O., Russell, R. \& Morizot, F. (2017). Facial contrast is a cross-cultural cue for perceiving age. Frontiers in Psychology, 8, 1-9.

Porcheron, A., Mauger, E. \& Russell, R. (2013). Aspects of facial contrast decrease with age and are cues for age perception. PLOS ONE, 8, e57985.

Richetin, J., Croizet, J. C. \& Huguet, P. (2004). Facial Make-up eclicits positive attitudes at the implicit level: Evidence from the implicit association test. Current Research in Social Psychology, 9, 145-164.

Russell, R., Kramer, S.S. \& Jones, A.L. (2017). Facial contrast declines with age but remains sexually dimorphic throughout adulthood. Adaptive Human Behavior and Physiology, 3, 293-303.

Russell, R., Porcheron, A., Sweda, J.R., Jones, A.L., Mauger, E. \& Morizot, F. (2016). Facial contrast is a cue for perceiving health from the face. Journal of Experimental Psychology: Human Perception and Performance, 42, 13541362.

Russell, R. (2009). A sex difference in facial pigmentation and its exaggeration by cosmetics. Perception, 38, 1211-1219.

Russell, R. (2003). Sex, beauty, and the relative luminance of facial features. Perception, 32, 1093-1107.

Stephen, I. D., Coetzee, V. \& Perrett, D. I. (2011). Carotenoid and melanin pigment coloration affect perceived human health. Evolution and Human Behavior, 32, 216-227.

Stephen, I. D., Coetzee, V., Law Smith, M. \& Perrett, D. I. (2009). Skin blood perfusion and oxygenation colour affect perceived human health. PloS One, 4, e5083. http://doi.org/10.1371/journal.pone.0005083

Stephen, I. D. \& McKeegan, A. (2010). Lip colour affects perceived sex typicality and attractiveness of human faces. Perception, 39, 1104-1110. http://doi.org/10.1068/p6730 
Todorov, A., Olivola, C. Y., Dotsch, R. \& Mende-Siedlecki, P. (2015). Social attributions from faces: Determinants, consequences, accuracy, and functional significance. Annual Review of Psychology, 66, 1-27.

Todorov, A., Mandisodza, A. N., Goren, A. \& Hall, C. C. (2005). Inferences of competence from faces predicts election outcomes. Science, 308, 16231626.

Vaillancourt, T. (2013). Do human females use indirect aggression as an intrasexual competition strategy. Philosophical Transactions of the Royal Society B, 368, http://dx.doi.org/10.1098/rstb.2013.0080.

Van Vugt, M., Hogan, R. \& Kaiser, R. B. (2008). Leadership, Followership, and Evolution: Some lessons from the past. American Psychologist, 63, 182-196.

Watkins, C. D. (2017). Creativity compensates for low physical attractiveness when individuals assess the attractiveness of social and romantic partners. Royal Society Open Science, 4, 160955.

Wildschut, T., Pinter, B., Vevea, J. L., Insko, C. A. \& Schopler, J. (2003). Beyond the group mind: A quantitative review of the interindividual-intergroup discontinuity effect. Psychological Bulletin, 129, 698-722.

Wilson, M. \& Daly, M. (2004). Do pretty women inspire men to discount the future? Proceedings of the Royal Society of London B, S177-S179.

Ungar, T., Martinetto, P., Ribarik, G., Dooryhee, E., Walter, P. \& Anne, M. (2002). Revealing the powdering methods of black makeup in Ancient Egypt by fitting microstructure based Fourier coefficients to the whole $x$-ray diffraction profiles of galena. Journal of Applied Physics, 91, 2455-2465. 